\title{
Cervico-Mediastinal Follicular Dendritic Cell Tumor: A Diagnostic and Therapeutic Challenge
}

\section{Rizzotto Giuseppe', La Rosa Filippo' ${ }^{1}$ and D'Ascanio Luca*}

${ }^{1}$ Department of Otolaryngology - Head \& Neck Surgery, Veneto Regional Cancer Center, Vittorio Veneto, Italy

'Department of Otolaryngology - Head \& Neck Surgery, "Carlo Poma" Civil Hospital, Mantova, Italy

\begin{abstract}
Background: Follicular Dendritic Cell (FDC) sarcoma is an extremely uncommon neoplasm with only few cases reported in the literature. The absolute rarity of FDC sarcoma with mediastinal localization has prevented the development of definite guidelines for the treatment of such tumor.

Methods and Results: We report the unique case of a 57 year-old-man with a cervico-upper mediastinum extensive $(64 \times 44 \mathrm{~mm})$ FDC sarcoma, recurred after multiple surgical and chemo-radiotherapeutical treatments, which was removed through anterior cervical approach without sternal-thoracotomy.

Conclusion: Immunohistochemistry is essential for FDC tumor differential diagnosis. Basing on its low-intermediate grade of malignancy, a minimally invasive and low-risk treatment, like cervical approach without sternal-thoracotomy, should be considered for the surgical removal of FDC sarcoma massively affecting the upper mediastinum.
\end{abstract}

Keywords: Follicular dendritic cell tumor; Surgery; Chemotherapy; Radiotherapy; Cervical approach

\section{Introduction}

Follicular Dendritic Cell (FDC) sarcoma, first reported in 1986, is a rare neoplastic proliferation of FDCs [1]. It origi $\neg$ nates from follicular dendritic cells of the lymphoid tissue and mainly affects lymph nodes. FDC sarcoma with extranodal origin is extremely rare, most commonly affecting the oral cavity, tonsil, gastrointestinal tract and liver [2]. The clinical-pathological characteristics and therapy of extranodal FDC sarcoma still remain under-recognized, mainly due to the limited number of cases reported in the literature [3]. In particular, definite evidences are lacking about the treatment options and overall prognosis of FDC sarcoma with mediastinal localization. We report the unique case of a cervico-upper mediastinum extensive FDC sarcoma, recurred after multiple surgical and chemotherapeutical treatments, which was removed through anterior cervical approach without sternal-thoracotomy. The reasons for this choice, in relation to FDC tumor site and clinical-pathological behavior, are discussed.

\section{Case Report}

A 57 year-old-man was admitted for the first time at the Department of Otolaryngology - Head \& Neck Surgery, Veneto Regional Cancer Center of Vittorio Veneto (Italy) for a long history of thoracic mass treated in 1987 with surgical removal through sternal-thoracothomy and subsequent chemotherapy (Cytoxan, Adriamycin, Oncovin) after a histological diagnosis of diffuse mixed B-cell lymphoma. In 1988, a 2 -centimeter thoracic residual mass was surgically removed. In 2001, the patient was submitted to a sternal-thoracothomy approach for the removal of a multilobulate thoracic mass with successive histological examination suggestive of follicular dendritic cell tumor. Because of a new thoracic FDC recurrence, in 2003 he was submitted to a left lateral thoracothomy approach for tumor removal. In 2004, a new anteriorposterior thoracothomy, followed by radiotherapy, was carried out for the persistence of FDC tumor in the para-aortic space. In 2007 the patient was submitted to an urgent tracheostomy because of acute dyspnea. Neck and chest Computed Tomography (CT) with contrast showed a new left mediastinal-laterocervical mass. Histological examination was positive for recurrence of FDC tumor and new chemotherapy was administered.

In 2009, a follow-up Computed Tomography (CT) spiral scan showed an enlargement of the left superior mediastinal space and lower neck with a $64 \times 44 \mathrm{~mm}$, multilobulate, para-aortic mass (Figures 1 and 2). Due to the chemo-radiotherapy treatments already performed with poor benefit and the numerous transthoracic approaches previously administrated in other hospitals, we decided to remove the mass by anterior cervical approach without sternal-thoracotomy. Patient's clinical history also included positivity for chronic $\mathrm{C}$ hepatitis and chronic ischemic heart disease.

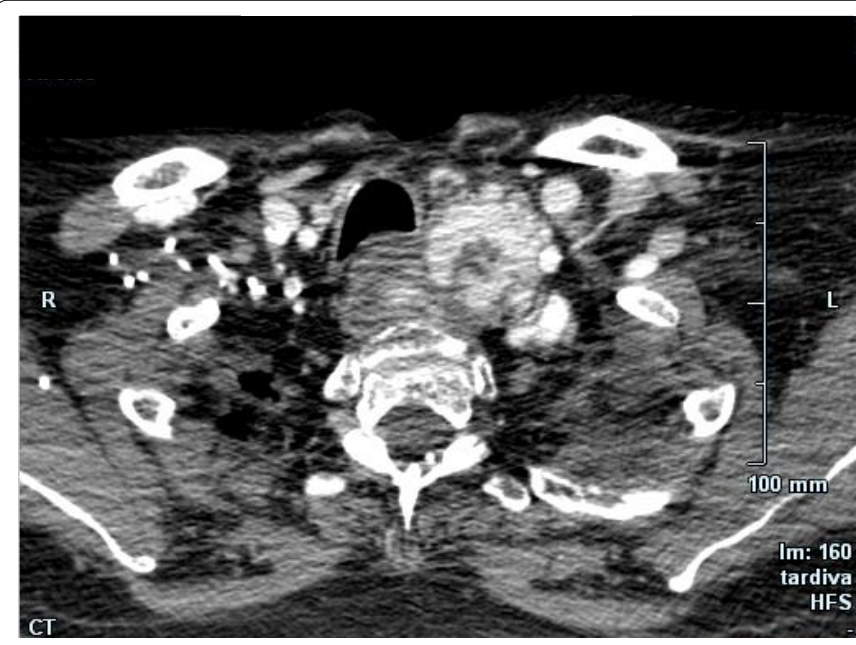

Figure 1: Axial CT spiral scan showing the enlargement of the left superior mediastinal space and lower neck with a $64 \times 44 \mathrm{~mm}$ multilobulate mass.

*Corresponding author: Luca D'Ascanio, Department of Otolaryngology - Head \& Neck Surgery, "Carlo Poma" Civil Hospital of Mantova, Strada Lago Paiolo 10-46100 Mantova, Italy, Tel: +393283186967; Fax: +39(0)376201091; E-mail: I.dascanio@gmail.com

Received February 17, 2014; Accepted March 20, 2014; Published April 05, 2014

Citation: Giuseppe R, Filippo LR, Luca DA (2014) Cervico-Mediastinal Follicular Dendritic Cell Tumor: A Diagnostic and Therapeutic Challenge. Surgery Curr Res 4: 185. doi: 10.4172/2161-1076.1000185

Copyright: @ 2014 Giuseppe R, et al. This is an open-access article distributed under the terms of the Creative Commons Attribution License, which permits unrestricted use, distribution, and reproduction in any medium, provided the original author and source are credited. 


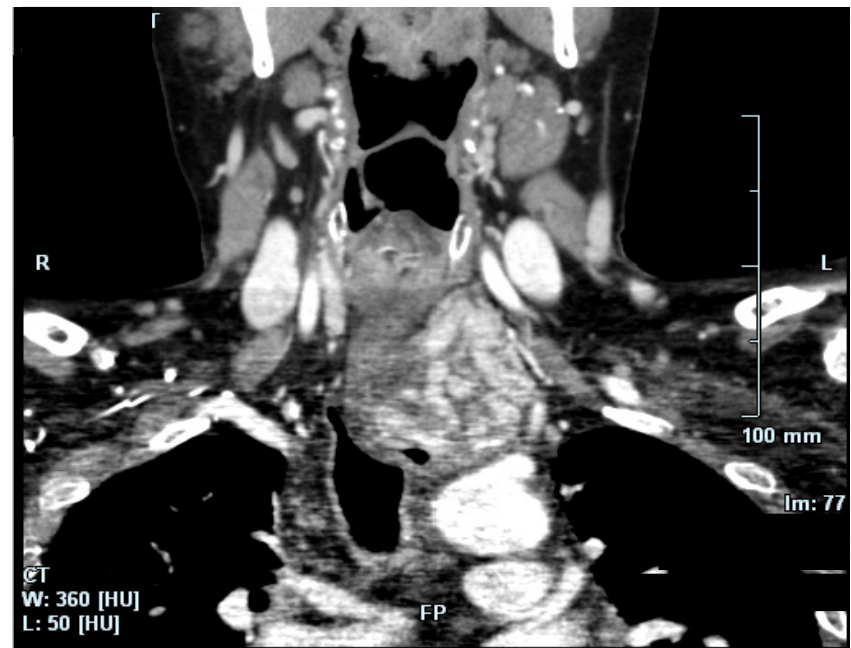

Figure 2: Coronal CT scan showing the cervico-superior mediastinal, paraaortic follicular dendritic cell sarcoma.

At surgery, the tan-white mass was identified in the upper mediastinum in proximity to the aortic artery with no evidence of either tissue or vascular invasion. The mass was resected in toto. Histopathologic assessment of the resected specimen showed matureappearing lymphocytes and spindle cells arranged in a haphazard fashion (Figure 3). Mitotic figures were not identified. Histological examination was positive for FDC sarcoma with immunohistochemical staining CD21+; CD23-; CD1a-; S100-; CD20-; CD3-; MPX-; CD34-; EMA- [4].

The patient spent 3 days at the intensive care unit of our Hospital for routine monitoring and then returned to our Department. After 4 years, the patient is alive and apparently disease-free (Figure 4). No further treatment has been performed.

\section{Discussion}

FDC is a rare tumor of dendritic-histiocytic cell origin, with only about 80 cases reported in the medical literature. Monda et al. first described FDC in 1986 and Chan et al reported the first extranodal location in 1994 [1,5]. About 30\% of FDC tumors have extranodal origin, i.e. palate, tonsils, stomach, mesocolon, pancreas, breast, small intestine, and spleen [2]. Primary FDC sarcoma of the upper mediastinum is extremely rare, with only 5 cases reported in the literature so far [6,7]. Even though it has been traditionally considered a low-grade neoplasm, a more aggressive behavior has been described, with a relevant frequency of recurrence (36\% of cases) and metastasis (25\%) [7]. A possible association with Epstein - Barr virus and Castelman's disease has also been reported [8].

Despite its frequent nodal origin, FDC tumors generally behave more like low-grade sarcomas than lymphomas [9]. Even though clinical and CT scan features (i.e. are the relative paucity of necrosis/ hemorrhage and the presence of coarse calcification) may help distinguishing FDC from bulky mediastinal masses such as lymphoma or small cell lung cancer, FDC sarcoma diagnosis can be awkward $[4,9,10]$. The differential diagnosis is based on immune histochemichal positivity to $\mathrm{CD} 21, \mathrm{CD} 23$, and $\mathrm{CD} 35$, along with podoplanin and cluster in $[4,9,10]$. The absence of immune histochemical stain was probably the cause of the likely wrong diagnosis (diffuse mixed B-cell lymphoma) performed in our patient in 1987.

Complete surgical resection is considered the treatment of choice.
Our experience confirms the scarce efficacy of adjuvant therapy (chemotherapy or radiation), probably because of the rarity of the lesion and the consequent lack of standard regimens. The prognosis of patients with follicular dendritic cell sarcoma is variable, mostly depending on surgical radicality [11]. Although parasternal mediastinotomy offers a good surgical exposure to most mediastinal masses, it can be associated with possible complications, such as pleural lesions and cardiac complications [11]. Our experience shows the necessity of preoperative immune histochemical examination for the differential diagnosis of FDC sarcoma and the appropriate treatment selection. In case of FDC tumor massively affecting the upper mediastinum, an anterior cervical approach allows to dominate and remove the soft encapsulated mass with a minimal risk of complications and less invasive approach with respect to sternal-thoracotomy. Tumor's capsule eases the dissection from the surrounding structures even after previous chemo-radiotherapy, as in our case. In relation to the high frequency of recurrence and low-grade of malignancy, we suggest performing a minimally invasive and low-risk treatment, like cervical

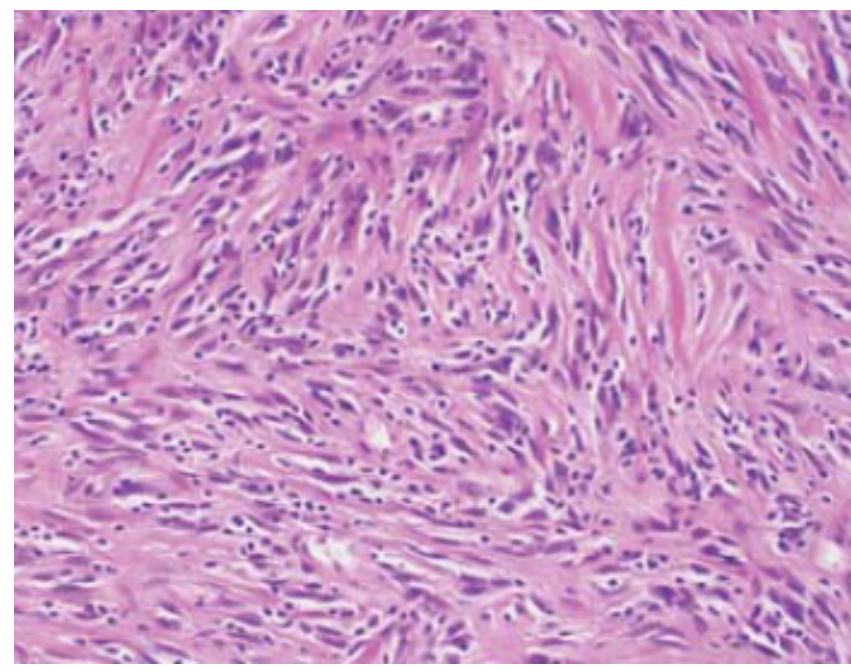

Figure 3: Hematoxylin \& Eosin stained slide (original magnification $\times 200$ ) showing matureappearing lymphocytes and spindle cells arranged in haphazard fashion with absent mitotic figures, which is consistent with follicular dendritic cell sarcoma.

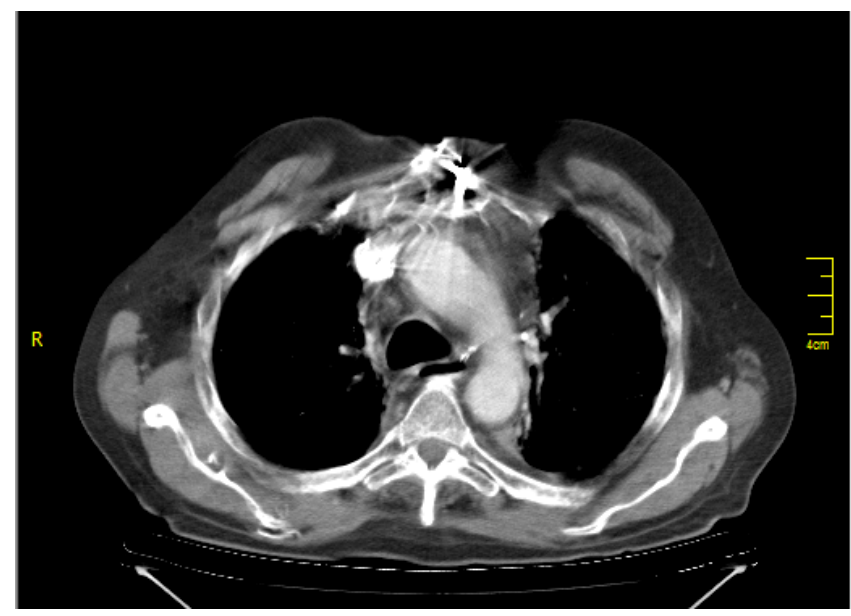

Figure 4: Four-year postoperative CT scan confirming the complete excision of the cervicosuperior mediastinal follicular dendritic cell sarcoma; no sign of recurrence can be noticed. 
Citation: Giuseppe R, Filippo LR, Luca DA (2014) Cervico-Mediastinal Follicular Dendritic Cell Tumor: A Diagnostic and Therapeutic Challenge. Surgery Curr Res 4: 185. doi:10.4172/2161-1076.1000185

Page 3 of 3

approach without sternal-thoracotomy, for the surgical removal of such tumor.

\section{References}

1. Monda L, Warnke R, Rosai J (1986) A primary lymph node malignancy with features suggestive of dendritic reticulum cell differentiation. A report of 4 cases. Am J Pathol 122: 562-572.

2. Shia J, Chen W, Tang LH, Carlson DL, Qin J, et al. (2006) Extranodal follicular dendritic cell sarcoma: clinical, pathologic, and histogenetic characteristics of an underrecognized disease entity. Virchows Arch 449: 148-158.

3. Li Z, Jin K, Yu X, Teng X, Zhou H, et al. (2011) Extranodal follicular dendritic cell sarcoma in mesentery: A case report. Oncol Lett 2: 649-652.

4. Malik A, Veniyoor A, Fanthome B, Dutta V (2012) Follicular dendritic cell sarcoma: a diagnostic challenge. J Cancer Res Ther 8: 306-307.

5. Chan JK, Tsang WY, Ng CS, Tang SK, Yu HC, et al. (1994) Follicular dendritic cell tumors of the oral cavity. Am J Surg Pathol 18: 148-157.
6. Kröber SM, Marx A, Aebert H, Dohmen BM, Kaiserling E (2004) Sarcoma of follicular dendritic cells in the dorsal mediastinum. Hum Pathol 35: 259-263.

7. Cano JR, Cerezo F, González A, Marchal T, Salvatierra A (2009) [Follicular dendritic cell tumour in the anterior mediastinum]. Cir Esp 85: 254-256.

8. Lin O, Frizzera G (1997) Angiomyoid and follicular dendritic cell proliferative lesions in Castleman's disease of hyaline-vascular type: a study of 10 cases. Am J Surg Pathol 21: 1295-1306.

9. Fonseca R, Tefferi A, Strickler JG (1997) Follicular dendritic cell sarcoma mimicking diffuse large cell lymphoma: a case report. Am J Hematol 55: 148-155.

10. Romero-Guadarrama MB, Reyes-Posada O, Hernández-González MM Durán-Padilla MA (2009) Follicular dendritic cell sarcoma/tumor: 2 cases of a rare tumor of difficult clinical and pathological diagnosis. Ann Diagn Pathol 13: $257-262$.

11. Burt M, Ihde JK, Hajdu SI, Smith JW, Bains MS, et al. (1998) Primary sarcomas of the mediastinum: results of therapy. J Thorac Cardiovasc Surg 115: 671-680. 